\title{
Correction to: Runs of homozygosity in sub-Saharan African populations provide insights into complex demographic histories
}

\author{
Francisco C. Ceballos ${ }^{1}\left(\mathbb{D} \cdot\right.$ Scott Hazelhurst ${ }^{1,2} \cdot$ Michèle Ramsay $^{1,3}$
}

Published online: 29 July 2019

○) Springer-Verlag GmbH Germany, part of Springer Nature 2019

\section{Correction to: Human Genetics}

https://doi.org/10.1007/s00439-019-02045-1

In the Original article published, the figure number 5: Genomic distribution of $\mathrm{ROH}$ is incorrectly published. The correct Fig. 5 is given below.

The original article can be found online at https://doi.org/10.1007/ s00439-019-02045-1.

Francisco C. Ceballos

ceballoscamina@gmail.com

1 Sydney Brenner Institute for Molecular Bioscience, Faculty of Health Sciences, University of the Witwatersrand, Johannesburg, South Africa

2 School of Electrical and Information Engineering, University of the Witwatersrand, Johannesburg, South Africa

3 Division of Human Genetics, School of Pathology, Faculty of Health Sciences, University of the Witwatersrand, Johannesburg, South Africa 


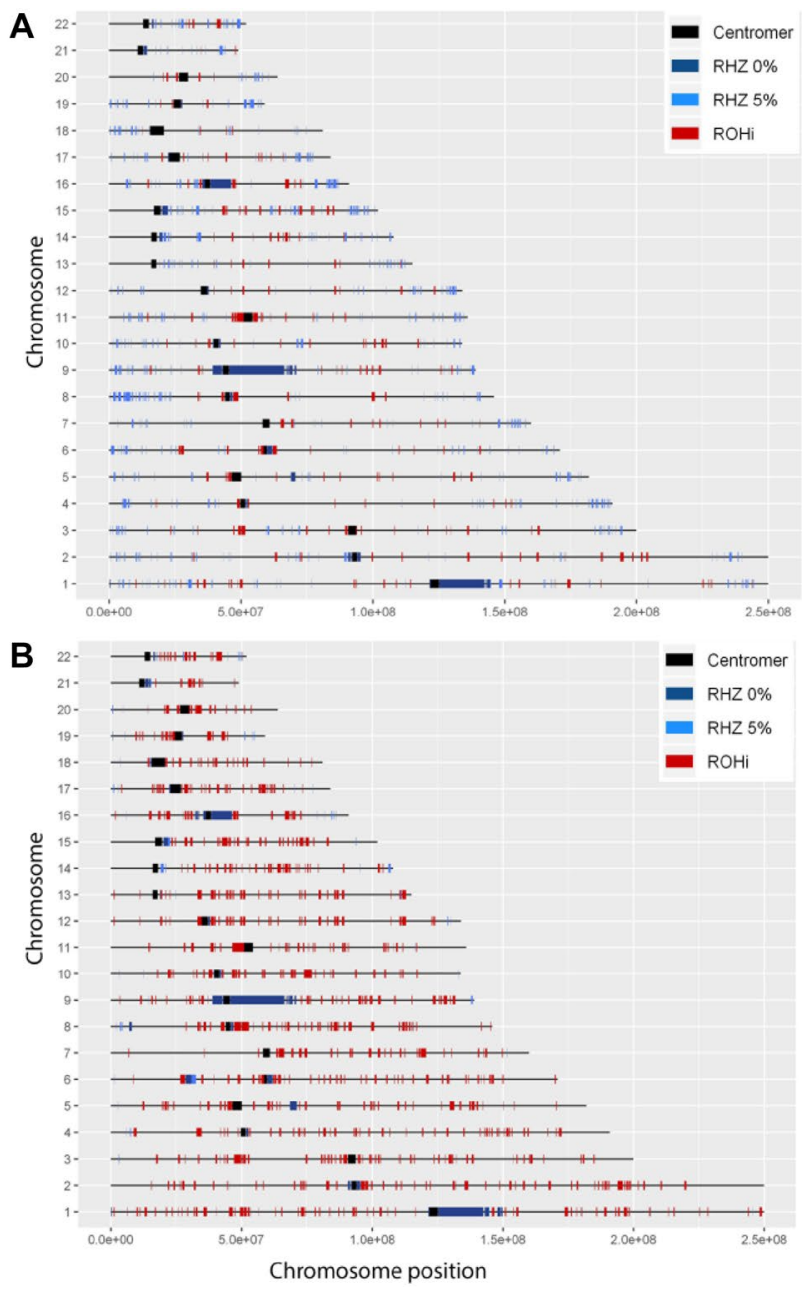

Fig. 5 Genomic distribution of ROH. Left side: genomic representation of the chromosomal location and size of runs of homozygosity islands (ROHi) and regions of heterozygosity (RHZ) for the Khoe and San (a) and European (b) regional groups. RHZ 0\%: genomic regions where no individual in the group has a $\mathrm{ROH}$. RHZ 5\%: genomic regions where $\leq 5 \%$ of the population has $\mathrm{ROH}$. Right side: heatmap and rooted dendrogram of the unique $\mathrm{ROH}$ islands (c) or RHZ (D) per geographical regional group and for admixed popula-

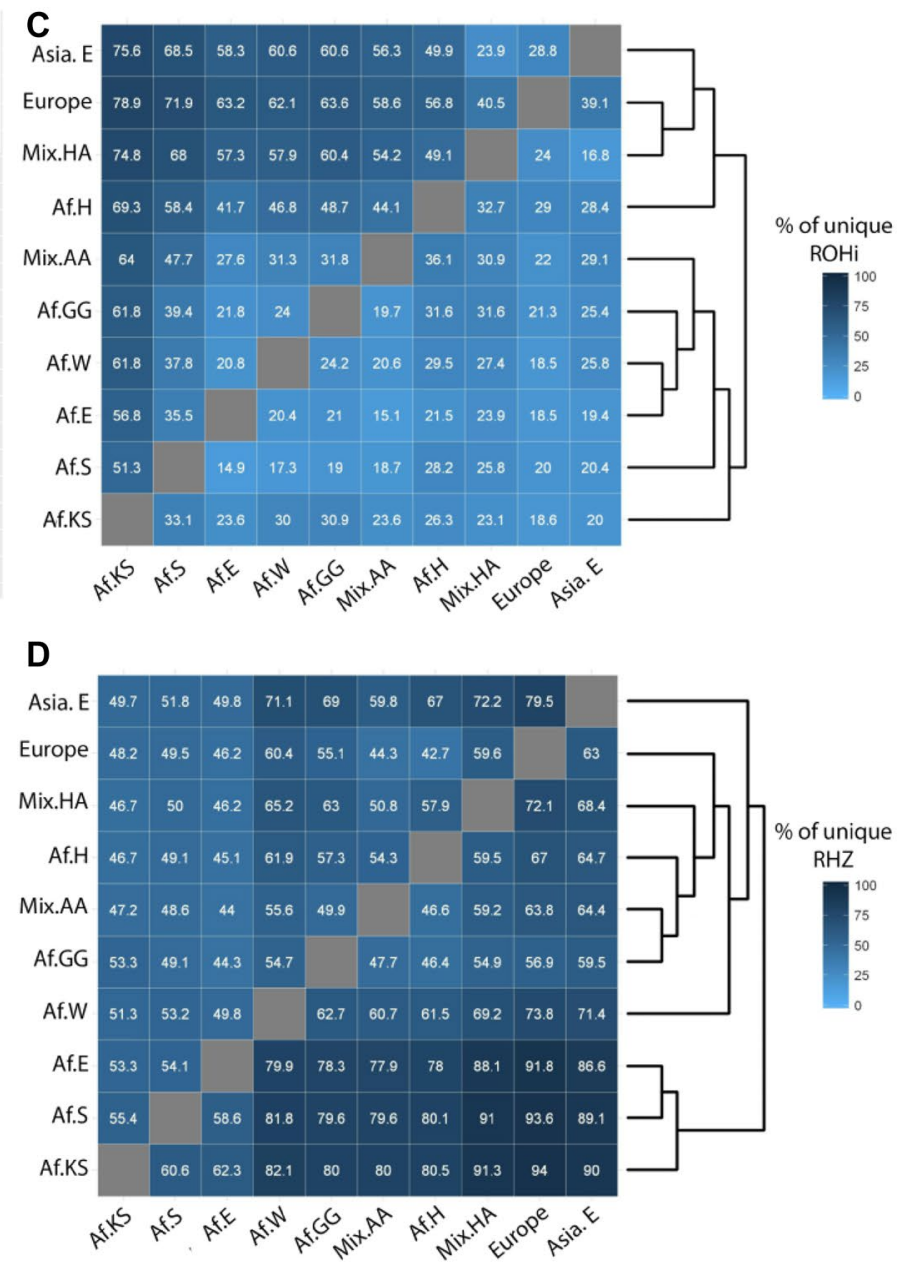

tions. The heatmap shows pairwise $\%$ of unique ROHi/RHZ between regional groups. The rooted dendrogram was obtained using optimal leaf ordering or OLO. Af.KS African Khoe and San populations, Af.S population from southern Africa, Af.E population from eastern Africa, $A f . W$ population from western Africa, $A f . G G$ population from the Gulf of Guinea, Mix.AA African-American admix populations, Mix.HA Hispanic-American admix populations, Europe European populations, Asia.E populations from eastern Asia

Publisher's Note Springer Nature remains neutral with regard to jurisdictional claims in published maps and institutional affiliations. 